\title{
Transmission Matrix and Faraday Rotation in a Structure Composed of Over-Dense Magnetized Plasma, Dielectric, and Prism Layers
}

\author{
M. Nejati AND L. RAJAeI* \\ Physics Department, Qom University, Qom, Iran \\ (Received May 21, 2016)
}

\begin{abstract}
In this study, the anomalous transmission of wave through over-dense magnetized plasma is investigated. Electromagnetic waves propagate in over-dense matter through an excitation of the surface waves. For excitation of the surface waves, the structure of the prism, dielectric and magnetized over-dense plasma are considered. Variations in the transmitted and reflected amplitudes with the incident angle $\theta$ is investigated. Moreover, the effect of the thicknesses of the dielectric and plasma layers and that of the plasma and cyclotron frequencies on the transmitted wave amplitude are studied. In addition, the Faraday rotation of the transmitted and reflected waves as functions of the incident angle is investigated. The effect of various parameters on the Faraday rotation is considered. The prism currently deployed in this structure decreases the restriction of dielectric constant and provides conditions for actual construction.
\end{abstract}

DOI: 10.12693/APhysPolA.130.1346

PACS/topics: 52.25.Xz, 52.59.Hq

\section{Introduction}

The study of the transmission of electromagnetic waves from normally opaque substances, such as over-dense plasmas, has attracted increasing attention because of its importance in many practical areas [1-6]. In the frequency region $\omega<\omega_{p}$, an over-dense plasma is completely opaque to electromagnetic waves. However, if surface plasmons are excited then electromagnetic waves can pass over-dense plasma [6-8]. The excitation of surface plasmons on the surface of the plasma only occurs in the presence of evanescent waves. Because the electromagnetic waves are bulk waves, they must be converted to evanescent waves in the direction perpendicular to the surface. There are various methods to convert a propagating wave into an evanescent one. This then allows the interaction of light with plasmons [9]. Welford et al. used the mechanism of total internal reflection of a near boundary inhomogeneity of the dielectric permittivity to provide the required evanescent waves [10]. Diffraction gratings can also be used to produce evanescent waves [11]. One of these methods is the use of a dielectric layer. The dielectric layer damped waves on both sides of the plasma which led to the excitation of the coupled plasmons on both sides of the over-dense plasma layer. The created coupled plasmons can then transfer the incident electromagnetic energy [6, 8, 12]. In our previous study, we used two dielectric layers, one on each side of a plasma, to produce evanescent waves [11]. However, to this end, the real part of the dielectric permittivity of the dielectric layer must be within the interval $0<\operatorname{Re} \varepsilon<1$. This has been shown in [6] and [12] by solving the dispersion relation.

\footnotetext{
*corresponding author; e-mail: 1-rajaei@qom.ac.ir
}

In this study, we add two prisms to our previous structure [11]. Because of the presence of a prism in this structure, the restriction on dielectric permittivity is removed. Furthermore, in our present paper, the over-dense plasma layer is assumed to be immersed in a steady, uniform homogeneous magnetic field. In this case, the equivalent dielectric constant of this layer is not merely a scalar function of frequency and converts to tensor. The nondiagonal components of this tensor cause rotation of the vertical component of the field, which is termed the Faraday rotation. The Faraday rotation describes the effect by which the electric field vector of propagating electromagnetic radiation rotates as it propagates through a magnetized plasma [13]. Much research effort has been concentrated on the Faraday rotation of electromagnetic waves. Lou et al. experimentally studied the Faraday rotation in high-pressure plasma [14]. The variance of the Faraday angle for magnetized plasma slabs at different anisotropy factors and the angle of inclination of prolate irregularities with respect to the external magnetic field was calculated by the perturbation method in Ref. [15].

In this paper, the structure consists of the magnetized over-dense plasma, dielectric layer, and prism is considered, and anomalous transmission of electromagnetic waves through this structure is observed. Moreover, the Faraday rotation of electromagnetic fields passing through over-dense magnetized plasma is investigated. This study is presented in four sections, including the Introduction as the first section. In Sect. 2, the theoretical model and anomalous transmission of electromagnetic waves through the considered structure is discussed. Moreover, the effects of various parameters on the transmitted and reflected wave's amplitudes are investigated. In Sect. 3, the Faraday rotation of the transmitted and reflected waves is presented. Finally, in Sect. 4, a summary and conclusion is given. 


\section{The model}

The configuration under consideration is shown in Fig. 1. This structure consists of a magnetized over-dense plasma layer with thickness $2 h_{1}$ protected by two dielectric layer with thickness $\left(h_{2}-h_{1}\right)$ and limited by two prisms.

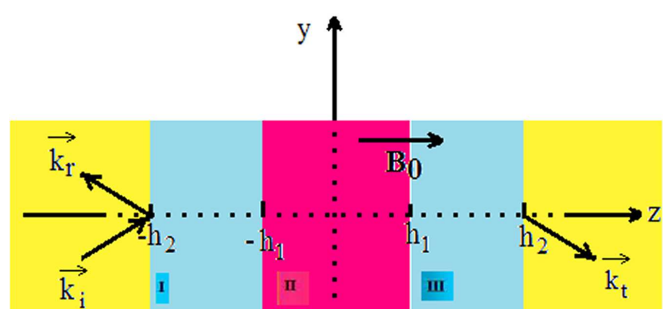

Fig. 1. Sketch of the structure consists of a magnetized over-dense plasma layer with thickness $2 h_{1}$ protected by two dielectric layer with thickness $\left(h_{2}-h_{1}\right)$ and limited by two prisms. The incident wave, the reflected wave, and the transmitted wave are respectively $k_{i}, k_{r}$ and $k_{t}$.

In these figures, the thickness is normalized by $k_{0}=$ $\omega / c$, where $\omega$ and $c$ are the frequency of the incident wave and the light velocity in vacuum, respectively. Furthermore, if the plasma is exposed to the external electromagnetic field, the dielectric tensor of the magnetized over-dense plasma is as follows [16]:

$$
\varepsilon=\left(\begin{array}{ccc}
\varepsilon_{1} & \mathrm{i} \varepsilon_{2} & 0 \\
-\mathrm{i} \varepsilon_{2} & \varepsilon_{1} & 0 \\
0 & 0 & \varepsilon_{3}
\end{array}\right),
$$

where

$$
\begin{aligned}
& \varepsilon_{1}=1-\frac{\omega_{p}^{2}}{\left(\omega^{2}-\omega_{c}^{2}\right)}, \\
& \varepsilon_{2}=\frac{\omega_{c} \omega_{p}^{2}}{\omega\left(\omega^{2}-\omega_{c}^{2}\right)}, \\
& \varepsilon_{3}=1-\frac{\omega_{p}^{2}}{\omega^{2}},
\end{aligned}
$$

where $\omega_{p}=4 \pi n \mathrm{e}^{2} / m_{e}$ and $\omega_{c}=e B_{0} / m_{e} c$ are the electron plasma frequency and the electron cyclotron frequency, respectively. Also, $n$ is the density of the electron, and $B_{0}$ is the external longitudinal magnetic field. In order to investigate the transmission of electromagnetic waves through the considered structure, we make use of a linearized set of cold-fluid-Maxwell equations,

$$
\begin{aligned}
& \boldsymbol{\nabla} \times \boldsymbol{B}=-\frac{4 \pi n_{0} e}{c} \boldsymbol{V}+\frac{1}{c} \frac{\partial}{\partial t}(\boldsymbol{E}), \\
& \boldsymbol{\nabla} \times \boldsymbol{E}=-\frac{1}{c} \frac{\partial \boldsymbol{B}}{\partial t}, \\
& \boldsymbol{\nabla} \cdot \boldsymbol{B}=0, \\
& \boldsymbol{\nabla} \cdot(\boldsymbol{E})=-4 \pi n e .
\end{aligned}
$$

and

$$
\frac{\partial \boldsymbol{V}}{\partial t}=-\frac{e}{m}\left(\boldsymbol{E}+\boldsymbol{V} \times \frac{\boldsymbol{B}_{0}}{c}\right) .
$$

Here $n_{0}$ and $n$ are the unperturbed and perturbed electron densities. For simplicity, the above equations be- come dimensionless and are redefined by the following assumptions:

$$
\left(\widetilde{\boldsymbol{r}}=k_{0} \boldsymbol{r}, \tilde{t}=\omega t\right) \quad \tilde{E}=\frac{\boldsymbol{E}}{E_{0}}, \quad \widetilde{\boldsymbol{B}}=\frac{\boldsymbol{B}}{B_{0}}
$$

In the following text, for simplicity we remove sign . By taking the time variation part of all field quantities as $\mathrm{e}^{-\mathrm{i} t}$ and finding the components of $\boldsymbol{v}$ from Eq. (9) and inserting them into Eq. (5), we find that

$$
\begin{aligned}
& \boldsymbol{\nabla} \times \boldsymbol{B}=-\mathrm{i} \varepsilon \boldsymbol{E}, \\
& \boldsymbol{\nabla} \times \boldsymbol{E}=i \boldsymbol{B},
\end{aligned}
$$

where $\varepsilon$ is the dielectric tensor for different environments. This tensor for the magnetized over-dense plasma is defined in Eq. (1). It should be noted that for the dielectric and prism regions the dielectric tensor are diagonal. By eliminating $B$ from Eqs. (11) and (12), we find that

$$
\boldsymbol{\nabla} \times \boldsymbol{\nabla} \times \boldsymbol{E}=\varepsilon \boldsymbol{E} .
$$

It is noticeable that the homogeneous magnetic field is in the direction $z\left(B_{0}=B_{0} \hat{k}\right)$, while the $z$ axis is perpendicular to the plasma layer and the incident wave is in the plane $(y, z)$. Therefore, the spatial part of the field components must be proportional to $\exp \left(i k_{y} y\right)$ with the coordinate $y$ running along the interface, and the wave amplitudes depend only on the variable $z$ and $k_{y}=\sin \theta$, where $\theta$ is the incident angle. By solving Eq. (13) in three directions, $x, y, z$, the components of the electric and magnetic fields in the plasma region are obtained by

$$
\begin{aligned}
& E_{x}(z)=E_{R_{1}} \mathrm{e}^{k_{R} z}+E_{R_{2}} \mathrm{e}^{-k_{R} z}+E_{L_{1}} \mathrm{e}^{k_{L} z} \\
& \quad+E_{L_{2}} \mathrm{e}^{-k_{L} z}, \\
& E_{y}(z)=a\left(E_{R_{1}} \mathrm{e}^{k_{R} z}+E_{R_{2}} \mathrm{e}^{-k_{R} z}\right) \\
& \quad+b\left(E_{L_{1}} \mathrm{e}^{k_{L} z}+E_{L_{2}} \mathrm{e}^{-k_{L} z}\right), \\
& \quad B_{x}(z)=\frac{-\mathrm{i} \varepsilon_{3}}{k_{y}^{2}-\varepsilon_{3}}\left[a k_{R}\left(E_{R_{1}} \mathrm{e}^{k_{R} z}+E_{R_{2}} \mathrm{e}^{-k_{R} z}\right)\right. \\
& \left.\quad+b k_{L}\left(E_{L_{1}} \mathrm{e}^{k_{L} z}+E_{L_{2}} \mathrm{e}^{-k_{L} z}\right)\right], \\
& B_{y}(z)=-\mathrm{i}\left[k_{R}\left(E_{R_{1}} \mathrm{e}^{k_{R} z}-E_{R_{2}} \mathrm{e}^{-k_{R} z}\right)\right. \\
& \left.\quad+k_{L}\left(E_{L_{1}} \mathrm{e}^{k_{L} z}-E_{L_{2}} \mathrm{e}^{-k_{L} z}\right)\right],
\end{aligned}
$$

where $E_{L_{i}}$ and $E_{R_{i}}$ are constants and $K_{R}, k_{L}, a$ and $b$ are defined as:

$$
\begin{aligned}
& K_{R}^{L}=\left[\frac{\left(\varepsilon_{1}+\varepsilon_{3}\right) k_{y}^{2} \pm \alpha}{2 \varepsilon_{3}}+\varepsilon_{1}\right]^{1 / 2}, \\
& \frac{b}{a}=\frac{i}{2 \varepsilon_{2} \varepsilon_{3}}\left[\left(\varepsilon_{1}-\varepsilon_{3}\right) k_{y}^{2} \pm \alpha\right]^{1 / 2},
\end{aligned}
$$

where

$$
\alpha=\left[\left(\varepsilon_{1}-\varepsilon_{3}\right) k_{y}^{4}-4 \varepsilon_{3} \varepsilon_{2}^{2} k_{y}^{2}+4 \varepsilon_{3}^{2} \varepsilon_{2}^{2}\right]^{1 / 2} .
$$

The field components in the dielectric region can be obtained by setting $\varepsilon_{1}=\varepsilon_{3}=\varepsilon_{d}$ and $\varepsilon_{2}=0$ in Eq. (1) and using Eq. (13) to find that

$$
\begin{aligned}
& E_{x}(z)=D_{1} \mathrm{e}^{k_{d} z}+D_{2} \mathrm{e}^{-k_{d} z}, \\
& E_{y}(z)=D_{3} \mathrm{e}^{k_{d} z}+D_{4} \mathrm{e}^{-k_{d} z},
\end{aligned}
$$




$$
\begin{aligned}
& B_{x}(z)=\frac{-\mathrm{i} \varepsilon_{d}}{k_{d}}\left(D_{3} \mathrm{e}^{k_{d} z}-D_{4} \mathrm{e}^{-k_{d} z}\right), \\
& B_{y}(z)=-\mathrm{i} k_{d}\left(D_{1} \mathrm{e}^{k_{d} z}-D_{2} \mathrm{e}^{-k_{d} z}\right),
\end{aligned}
$$

where $k_{d}=\left(k_{y}^{2}-\varepsilon_{d}\right)^{1 / 2}$ and $D_{i}^{\prime}$ s are arbitrary constants. By considering the dielectric tensor for the prism region can be obtained by setting $\varepsilon_{1}=\varepsilon_{3}=\varepsilon_{p}$ and $\varepsilon_{2}=0$ in Eq. (1) and the solution to Eq. (13) in the prism region, components of the electric and magnetic fields are obtained. The radiated wave in the prism region $\left(z<-h_{2} / k_{0}\right)$ has the following magnetic and electric components:

$$
\begin{aligned}
& E_{x}(z)=E_{0 x} \mathrm{e}^{\mathrm{i} z \sqrt{\varepsilon_{p}} \cos \theta}+R_{x} \mathrm{e}^{-\mathrm{i} z \varepsilon_{p} \cos \theta}, \\
& E_{y}(z)=E_{0 y} \mathrm{e}^{\mathrm{i} z \sqrt{\varepsilon_{p}} \cos \theta}+R_{y} \mathrm{e}^{-\mathrm{i} z \varepsilon_{p} \cos \theta}, \\
& B_{x}(z)=\frac{-1}{\cos \theta}\left(E_{0 y} \mathrm{e}^{\mathrm{i} z \sqrt{\varepsilon_{p}} \cos \theta}-R_{y} \mathrm{e}^{-\mathrm{i} z \varepsilon_{p} \cos \theta}\right), \\
& B_{y}(z)=\cos \theta\left(E_{0 x} \mathrm{e}^{\mathrm{i} z \sqrt{\varepsilon_{p}} \cos \theta}-R_{x} \mathrm{e}^{-\mathrm{i} z \varepsilon_{p} \cos \theta}\right),
\end{aligned}
$$
and in region $z>h_{2} / k_{0}$ has the following form:

$$
\begin{aligned}
& E_{x}(z)=T_{0 x} \mathrm{e}^{\mathrm{i} z \sqrt{\varepsilon_{p}} \cos \theta}, \\
& E_{y}(z)=T_{0 y} \mathrm{e}^{\mathrm{i} z \sqrt{\varepsilon_{p}} \cos \theta}, \\
& B_{x}(z)=\frac{-1}{\cos \theta} T_{0 y} \mathrm{e}^{\mathrm{i} z \sqrt{\varepsilon_{p}} \cos \theta}, \\
& B_{y}(z)=\cos \theta T_{0 x} \mathrm{e}^{\mathrm{i} z \sqrt{\varepsilon_{p}} \cos \theta} .
\end{aligned}
$$

By applying the boundary conditions, i.e., continuity of $E_{x}, E_{y}, B_{x}$, and $B_{y}$ at all the boundaries of Fig. $1, R_{x}$, $R_{y}, T_{x}$ and $T_{y}$ refer to the components of the reflection and transmission electromagnetic wave, we obtain a system of sixteen equations from which we create a transition matrix. In the following, by use of the transition matrix, we investigate the resulting expressions for the transmitted and reflected coefficients as functions of the incident angle of the wave, the plasma density, the thicknesses of both dielectric and plasma layers...

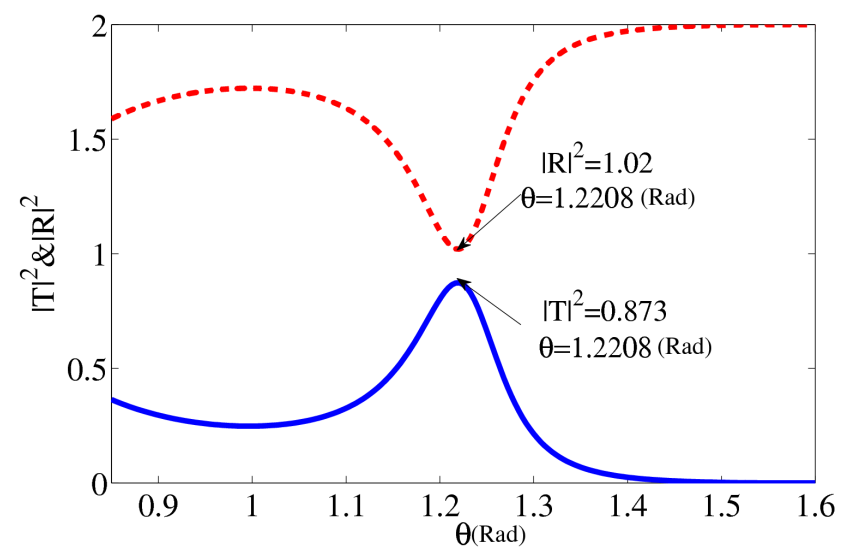

Fig. 2. The transmission amplitude (solid line) and the reflection amplitude (dashed line), as functions of the incident angle $\theta$. The parameters of the thickness of the plasma and the dielectric layers, the plasma and the cyclotron frequencies, respectively, are $h_{1}=0.086$, $h_{2}=2.11, \omega_{p} / \omega=4$ and $\omega_{c} / \omega=0.001$.

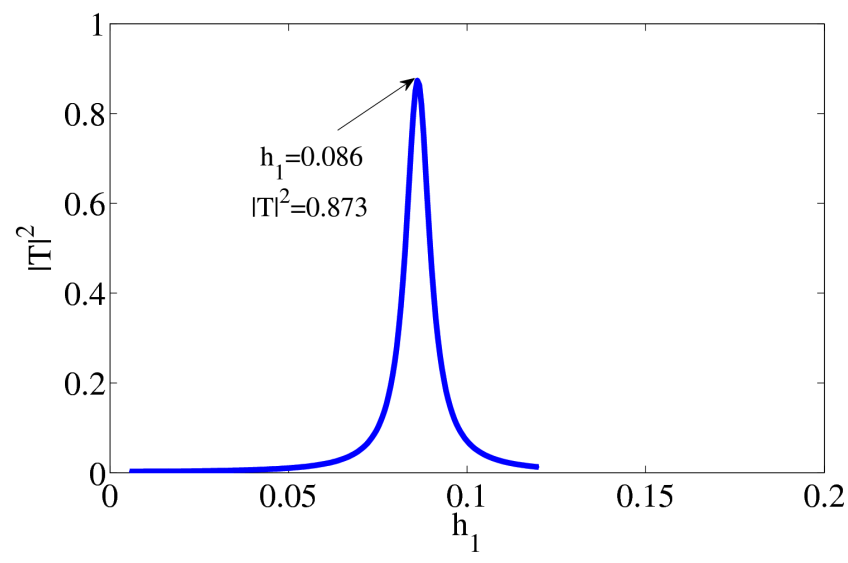

Fig. 3. The transmission amplitude as a function of the width of the plasma layer. The thickness of the dielectric layer, the incident angle, the plasma frequency and the cyclotron frequency, respectively, are $h_{2}=2.11$, $\theta=1.2208 \mathrm{rad}, \omega_{p} / \omega=4$, and $\omega_{c} / \omega=0.001$.

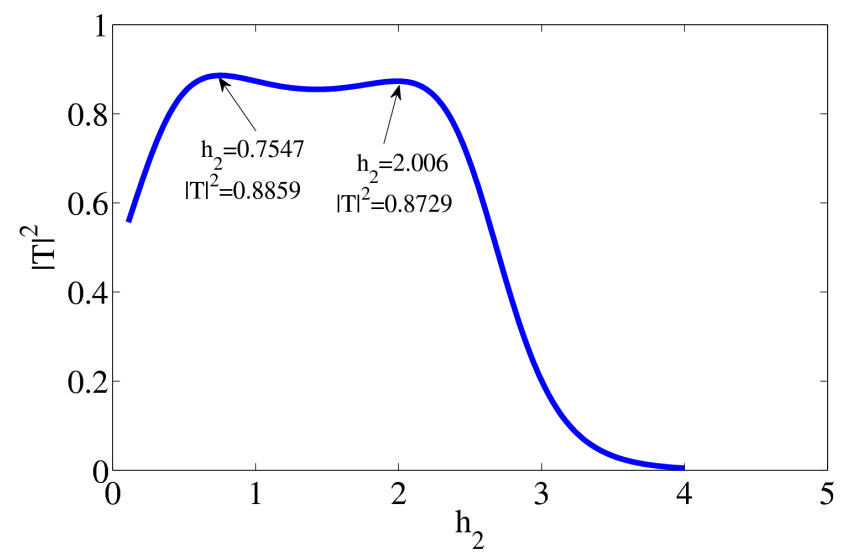

Fig. 4. The transmission amplitude as a function of the width of the dielectric layer. The thickness of the plasma layer, the incident angle, the plasma frequency and the cyclotron frequency, respectively, are $h_{1}=0.086, \theta=$ $1.2208 \mathrm{rad}, \omega_{p} / \omega=4$, and $\omega_{c} / \omega=0.001$.

Variations in the transmitted and reflected amplitudes with the incident angle $\theta$ are plotted in Fig. 2. The solid and dashed lines represent the transmission coefficient $\left(|T|^{2}=\left|T_{x}\right|^{2}+\left|T_{y}\right|^{2}\right)$ and the reflection coefficient $\left(|R|^{2}=\left|R_{x}\right|^{2}+\left|R_{y}\right|^{2}\right)$, respectively. The transmission coefficient has its maximum value at an incident angle of $\theta=1.2208 \mathrm{rad}$ and reflection coefficient has its minimum value at this angle. The other parameters in Fig. 2 are $h_{1}=0.086, h_{2}=2.11, \omega_{p} / \omega=4$ and $\omega_{c} / \omega=0.001$, $\varepsilon_{p}=2.295$ and $\varepsilon_{d}=1.2$.

Figure 3 represents a transmission coefficient $|T|^{2}$ as a function of the thickness of the plasma layer $h_{1}$. It shows that for a fixed incident angle $\theta=1.2208 \mathrm{rad}$, transmission coefficient has one peak at $h_{1}=0.0861$. The other parameters in this figure are the same as in Fig. 2.

The effects of the thickness of the dielectric layer on the transmission properties of the slab are shown in Fig. 4. 


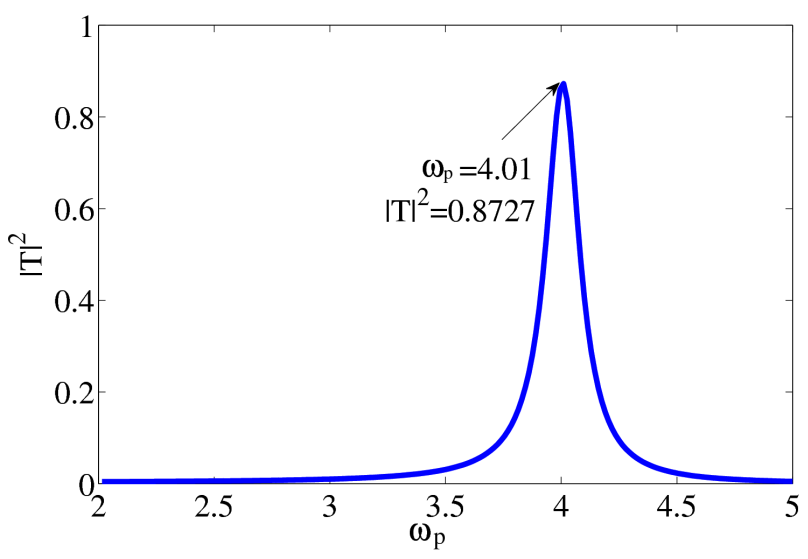

Fig. 5. The transmission amplitude as a function of the plasma frequency. The parameters of the thickness of the plasma and the dielectric layers and the cyclotron frequency and the incident angle, respectively are $h_{1}=$ $0.086, h_{2}=2.11, \omega_{c} / \omega=0.001$ and $\theta=1.2208 \mathrm{rad}$.

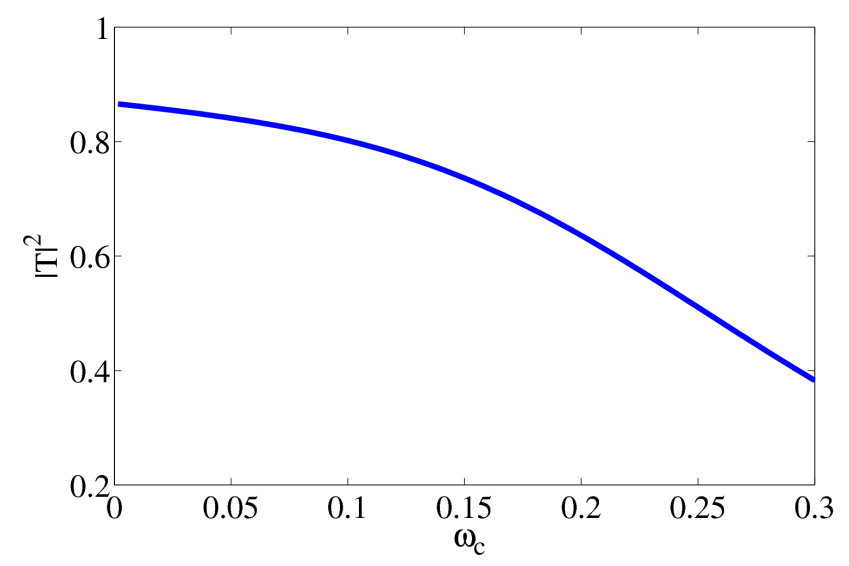

Fig. 6. The transmission amplitude as a function of the cyclotron frequency. The parameters of the incident angle, the thickness of the plasma and the dielectric layers and the plasma frequency, respectively are $\theta=$ $1.2208 \mathrm{rad}, h_{1}=0.086, h_{2}=2.11$ and $\omega_{p} / \omega=4$.

The transmission coefficient $|T|^{2}$ has two peaks, at $h_{2}=$ 0.75 and $h 2=2$.

Figure 5 illustrates $|T|^{2}$ as a function of plasma frequency $\omega_{p} / \omega$, and it can be seen that the transmission coefficient $|T|^{2}$ contains a maximum at $\omega_{p} / \omega=4.01$.

Figure 6 represents the transmission coefficient $|T|^{2}$ as a function of cyclotron frequency $\omega_{c} / \omega$. It shows that, for a fixed set of geometric parameters, the transmission coefficient $|T|^{2}$ is decreased by increasing the cyclotron frequency. In comparison to our previous study, it can be obtained that transmission properties of the slab have two maxima at incident angles of $\theta=0.88 \mathrm{rad}$ and $\theta=2.28 \mathrm{rad}$ while in the presence of prism, the transmission coefficient has one peak at an incident angle of $\theta=1.2208 \mathrm{rad}$. Moreover, as can be seen in our previous study, maxima of transmission coefficient occur at $h_{1}=0.087, h_{2}=0.21, \omega_{p} / \omega=1.6$ and $\omega_{c} / \omega=0.001$ that is different to conditions in this study.

\section{Faraday rotation effect}

As shown in the previous section, in the presence of a magnetic field, the permittivity tensor of over-dense plasma convert to a non-diagonal tensor. The nondiagonal components of this tensor cause rotation of the vertical component of the field, and this is termed Faraday rotation. As regards to over-dense plasmas, they have negative permittivity in $\left(\omega<\omega_{p}\right)$ and are typically opaque [17]. However, the electromagnetic waves are transmitted through the over-dense plasma only at resonant conditions of the excitation of the surface wave modes. The calculations in our previous paper [11] show that the presence of dielectric layers with $0<\operatorname{Re} \varepsilon<1$ can provide these resonant conditions [12]. In this study, by adding two prisms to our previous structure, the restriction $0<\operatorname{Re} \varepsilon<1$ is removed. First, we studied the manifestation of the Faraday rotation of the electromagnetic waves passing through the entire structure of Fig. 1. As mentioned earlier, when the wave is incident to the slab, an amount of their energy is transmitted, and another amount is reflected. By calculating the $y$ component with respect to the $x$ component of transmission, the Faraday rotation for the transmitted wave is obtained

$\tan \phi=\left|T_{y} / T_{x}\right|$,

and the Faraday rotation for reflected wave is

$$
\tan \phi=\left|R_{y} / R_{x}\right| \text {, }
$$

where $T_{x}, T_{y}, R_{x}$ and $R_{y}$ are $x$ and $y$ components of transmission $T$ and reflection $R$, respectively. Figures $7-$ 10 show the results of the Faraday rotation for the wave transmitted and reflected of the slab of Fig. 1. These figures demonstrate the angle of rotation of the electric field, $\phi-\pi / 4$, where $\pi / 4$ represents the angle between the components of the incident electric field, because of $\left|E_{x} / E_{y}\right|=1$.

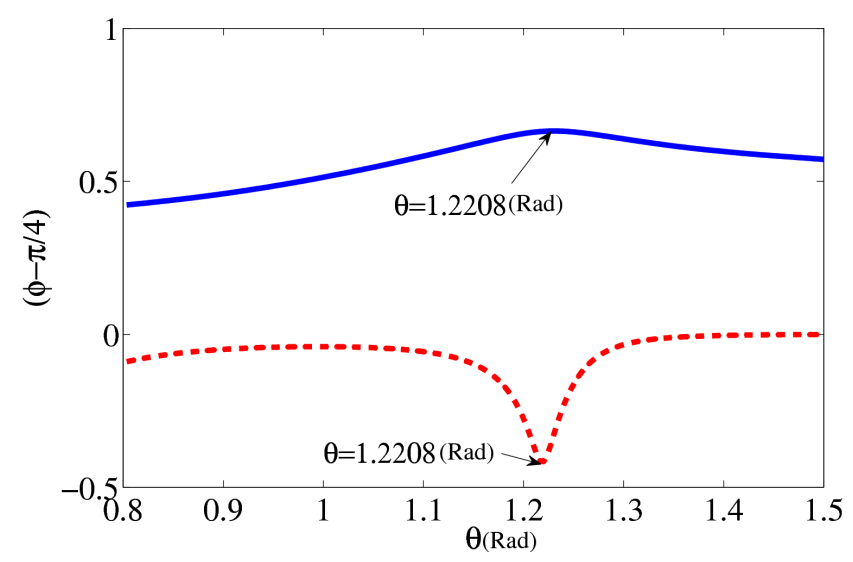

Fig. 7. The Faraday rotation of the transmitted wave (solid line) and the reflected wave (dashed line) as functions of the incident angle $\theta$. The parameters of the thickness of the plasma and the dielectric layers, the plasma and the cyclotron frequencies, respectively, are $h_{1}=0.086, h_{2}=2.11, \omega_{p} / \omega=4$ and $\omega_{c} / \omega=0.001$.

In Fig. 7 , the angle of rotation of the electric field $\phi-\pi / 4$ as a function of the incident angle $\theta$ is plotted. 


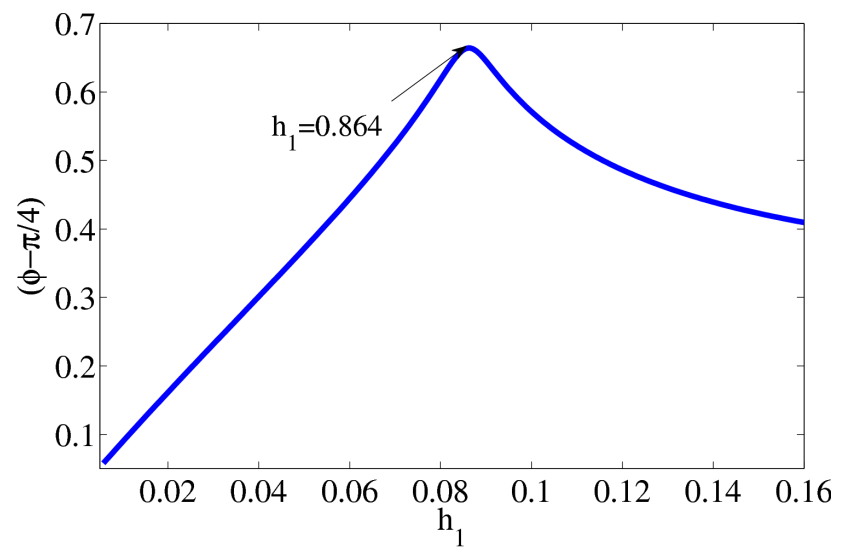

Fig. 8. The Faraday rotation of the transmitted wave as function of thickness of the plasma layer. The parameters of the incident angle, the thickness of the dielectric layer, the plasma and the cyclotron frequencies respectively are $\theta=1.2208 \mathrm{rad}, h_{2}=2.11, \omega_{p} / \omega=4$ and $\omega_{c} / \omega=0.001$.

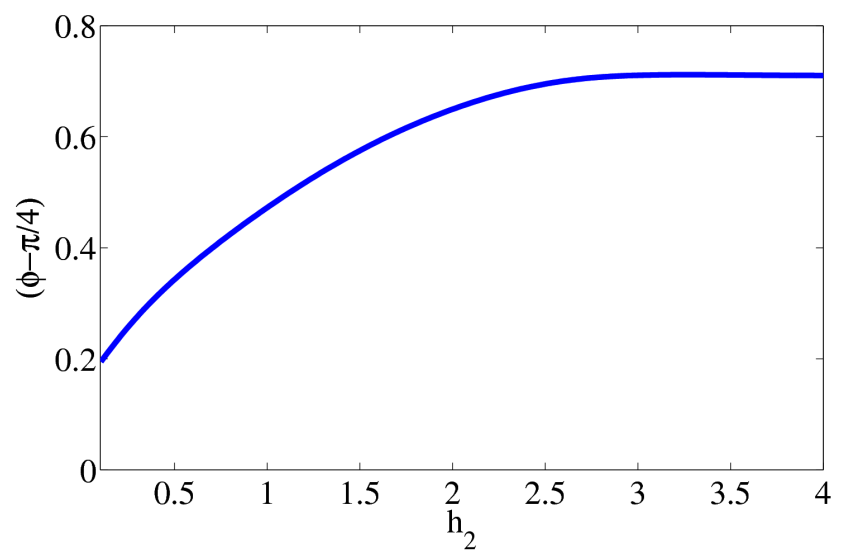

Fig. 9. The Faraday rotation of the transmitted wave as function of thickness of the dielectric layer. The parameters of the incident angle, the thickness of the plasma layer, the plasma and the cyclotron frequencies respectively are $\theta=1.2208 \mathrm{rad}, h_{1}=0.086, \omega_{p} / \omega=4$ and $\omega_{c} / \omega=0.001$.

The solid and dashed lines represent the rotation angle for the transmitted and reflected waves, respectively. According to Fig. 7, for $h_{1}=0.086, h_{2}=2.11, \omega_{p} / \omega=4$, $\omega_{c} / \omega=0.001$, and $\varepsilon_{p}=2.295$, the maximum of the rotation angle for the transmitted wave occurs exactly at those incident angles at which the transmitted amplitude obtains its maximum value. Specifically at $\theta=$ $1.2208 \mathrm{rad}$, the minimum rotation angle for the reflected wave occurs at the same angle $\theta=1.2208 \mathrm{rad}$. As shown in Fig. 2, the reflection amplitude is at its minimum value at this angle. These results show that Faraday rotation can occur in over-dense magnetized plasma for both reflected and transmitted waves. Figure 8 shows the angle of Faraday rotation $(\phi-\pi / 4)$ as a function of thickness of the plasma layer $h_{1}$. It can be seen that the rotation angle for the transmitted wave contains a maximum at

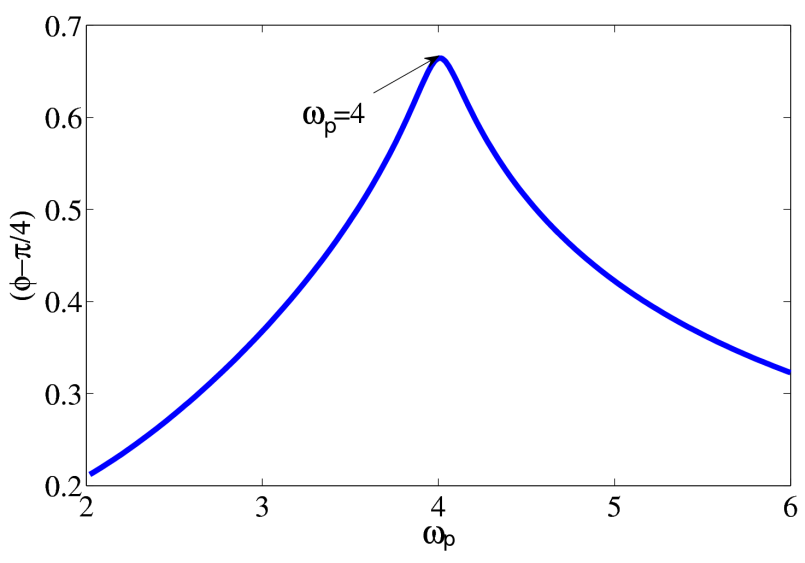

Fig. 10. The Faraday rotation of the transmitted wave as function of plasma frequency. The parameters of the incident angle, the thickness of the plasma and the dielectric layers, the cyclotron frequency respectively are $\theta=1.2208 \mathrm{rad}, h_{1}=0.086, h_{2}=2.11$ and $\omega_{c} / \omega=0.001$.

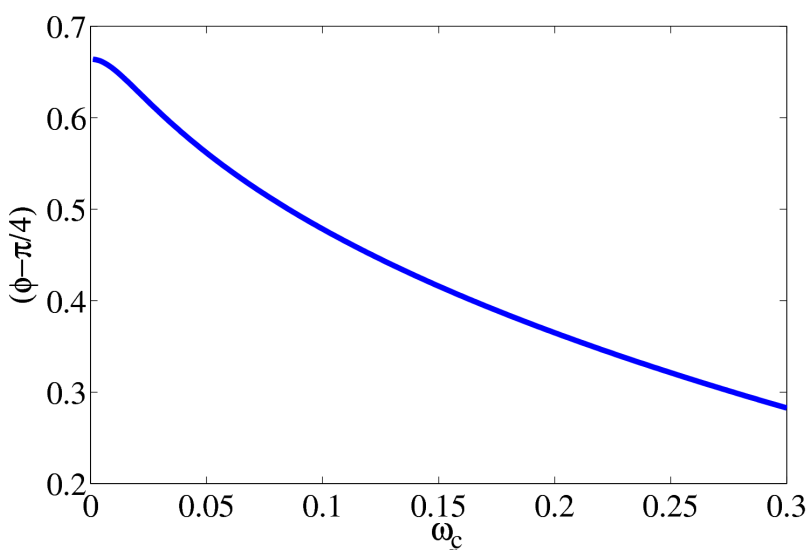

Fig. 11. The Faraday rotation of the transmitted wave as function of cyclotron frequency. The parameters of the incident angle, the thickness of the plasma and the dielectric layers, the plasma frequency, respectively, are $\theta=1.2208 \mathrm{rad}, h_{1}=0.086, h_{2}=2.11$ and $\omega_{p} / \omega=4$.

$h_{1}=0.086$. The other parameters in this figure are the same as in Fig. 7. According to Figs. 3 and 8, we find that the maximum of the rotation angle for the transmitted wave occurs at exactly the thicknesses of the plasma layer at which the transmitted wave amplitude obtains its maximum value.

The effect of the width of the dielectric layer on the angle of Faraday rotation is shown in Fig. 9. As shown in this figure, the angle of Faraday rotation increases by increasing the widths of the dielectric layer and approaches a constant value at approximately $h_{2}<3$.

Figure 10 illustrates the amount of transmitted wave Faraday rotation with respect to the plasma frequencies. It shows that the angle of the Faraday rotation increases with the plasma frequency and reaches a maximum value at $\omega_{p}=4$ and then decreases by increasing $\omega_{p}$. Finally, the effect of the cyclotron frequency on the 
angle of Faraday rotation is plotted in Fig. 11. This figure shows that the angle of Faraday rotation decreases by increasing cyclotron frequency $\omega_{c}$. The other parameters in Figs. 10 and 11 are the same as in Fig. 7. In comparison to our previous study, we observe that, in the absence of the prism, the maximum Faraday rotation angles for the transmitted wave occur at the incident angles which correspond to the maximum transmitted amplitudes, namely $\theta=0.88 \mathrm{rad}$ and $\theta=2.3 \mathrm{rad}$ [11], but while in presence of a prism, the Faraday rotation for the transmitted wave has only one peak, at $\theta=1.2208 \mathrm{rad}$.

\section{Conclusions}

This article was devoted to the study of the Faraday rotation of electromagnetic waves passing through overdense plasma. To this end, cold fluid Maxwell equations and continuous conditions of tangential components of electric and magnetic fields were used to calculate transmission and reflection coefficients. Furthermore, we investigated the Faraday rotation of electromagnetic fields passing through over-dense magnetized plasma. It has been shown that the Faraday rotation can occur in overdense magnetized plasma for both reflected and transmitted waves. In addition to the effect of the wave incident angle, the thicknesses of both dielectric and plasma layers and the plasma and cyclotron frequencies on Faraday rotation of the transmitted wave were investigated. The investigations showed that the Faraday rotation for transmission waves is at its maximum under exactly the same conditions where high-transparency can be observed. In the structure that was introduced in this article, the dielectric layer was proposed as a diffracting layer, which is required to produce the surface waves. In the absence of the prism, it is necessary to use a dielectric with $0<\operatorname{Re} \varepsilon<1$. This has been shown in [6] and [12] by solving the dispersion relation. Because of the presence of a prism in this structure, the restriction $0<\operatorname{Re} \varepsilon<1$ has been removed.

\section{References}

[1] S. Xi, Phys. Rev. Lett. 103, 194801 (2009).

[2] V.I. Slyusar, in: Int. Conf. on Antenna Theory and Techniques, Lviv, Lviv 2009.

[3] M. Beruete, M. Navarro-CĂa, M. Sorolla, I. Campillo, Opt. Expr. 16, 9677 (2008).

[4] R. Merlin, Appl. Phys. Lett. 84, 1290 (2004).

[5] Y.H. Chen, G.Q. Liang, J.W. Dong, H.Z. Wang, Phys. Lett. A 351, 446 (2006).

[6] L. Rajaei, S. Miraboutalebi, B. Shokri, Phys. Scr. 84, 015506 (2011).

[7] Yu.P. Bilokh, J. Felsteiner, Y.Z. Slutsker, Phys. Rev. Lett. 95, 165003 (2005).

[8] S. Miraboutalebi, L. Rajaei, M.K. Khadivi Borogeni, J. Theor. Appl. Phys. 7, 1 (2013).

[9] K.Y. Bliokh, Y.P. Bliokh, V. Freilikher, S. Savelev, F. Nori, Rev. Mod. Phys. 80, 1201 (2008).

[10] K.R. Welford, J.R. Sambles, J. Mod. Opt. 35, 1467 (1988).

[11] L. Rajaei, S. Miraboutalebi, M. Nejati, in: Contrib. Plasma Phys. 55, 513 (2015).

[12] R. Dragila, B. Luther-Davies, S. Vukovic, Phys. Rev. Lett. 55, 1117 (1985).

[13] K. Asada, M. Inoue, Y. Uchida, S. Kameno, K. Fujisawa, S. Iguchi, M. Mutoh, Publ. Astron. Soc. J. 54, L39 (2002).

[14] S.Q. Luo, J.E. Scharer, M. Thiyagarajan, C.M. Denning, IEEE Trans. Plasma Sci. 34, (2006).

[15] G.V. Jandieri, A. Ishimaru, V.G. Jandieri, N.N. Zhukava, Progr. Electromagn. Res. 112, 63 (2011).

[16] N.A. Krall, A.W. Trivelpiece, Principles of Plasma Physics, McGraw-Hill, 1973.

[17] D.R. Smith, W.J. Padilla, D.C. Vier, S.C. NematNasser, S. Schultz, Phys. Rev. Lett. 84, 4184 (2000). 